\title{
Thromboprophylaxis in women undergoing gynecological surgery or assisted reproductive techniques: new advances and challenges
}

\author{
Magdalena Piróg ${ }^{1}$, Robert Jach ${ }^{1}$, Anetta Undas ${ }^{2}$ \\ ${ }^{1}$ Department of Gynecological Endocrinology, Jagiellonian University Medical College, Krakow, Poland \\ ${ }^{2}$ Institute of Cardiology Jagiellonian University Medical College, and John Paul II Hospital, Krakow, Poland
}

\begin{abstract}
Venous thromboembolism (VTE) affects $0.1-0.3 \%$ people each year. The risk of VTE following gynecological surgery and invasive procedures is well established and presents one of the major challenges in gynecological practice. Moreover, commonly use assisted reproductive techniques (ART) are reported to increase the risk of VTE. Despite continued efforts to reduce its incidence, postoperative VTE remains the second most common perioperative complication and the third most common cause of mortality.

Several practice guidelines have been developed regarding prophylaxis and treatment of VTE. However, there is a large inconsistency between the recommendations and the medical practice in various centers. Moreover, prophylaxis in gynecological patients and women undergoing ART should be chosen individually for the patient, taking into account the possible risk factors for VTE and perioperative bleeding complications. Until recently, the percentage of women, who have not received anticoagulant prophylaxis or administrated it inadequately may reach $50 \%$.

This paper presents the current recommendations regarding thromboprophylaxis in women undergoing gynecological surgery or assisted reproductive techniques and addresses challenging practical issues in this field.
\end{abstract}

Key words: thromboprophylaxis, venous thromboembolism, hysterectomy, ovarian cancer, assisted reproductive techiques

Ginekologia Polska 2016; 87, 11: 773-779

\section{INTRODUCTION}

Venous thromboembolism (VTE), i.e. deep vein thrombosis (DVT) and pulmonary embolism (PE), affect 1 to 3 per 1000 persons per year [1]. It is estimated that in Poland DVT is diagnosed in approximately 50,000 people per year, while 20,000 patients per year suffer from PE [1]. Pulmonary embolism leads to death in $30 \%$ of the patients, while a proper anticoagulation therapy reduces PE-related mortality to $2-8 \%[2,3]$.

Surgery and invasive procedures are the well established risk factors of VTE. Despite continued efforts to reduce its incidence, postoperative VTE remains the second most common perioperative complication and the third most common cause of mortality [4]. The risk of VTE depends on genetic and environmental factors such as age, sex, thrombophilia, history of VTE, malignancy or hormone therapy, all these risk factors may additionally increase the VTE risk in perioperative period [5].

Surgical gynecological patients are at a particularly high risk of developing VTE [6]. Although several practice guidelines have been developed regarding prophylaxis and treatment of VTE, there is a large inconsistency between the recommendations and the medical practice in various centers. Even though many strategies have been proposed to improve the effectiveness and safety of VTE prophylaxis and treatment, it remains unsatisfactory [7].

Moreover, recommendations of VTE prophylaxis issued by the Polish Gynecological Society differ from guidelines recommended by other societies [8].

Therefore, this review article presents the current recommendations regarding thromboprophylaxis in women undergoing gynecological surgery or assisted reproductive 
techniques and addresses challenging practical issues in this field.

\section{THROMBOPROPHYLAXIS IN GYNECOLOGY}

The prevalence of VTE after gynecologic surgery varies from $15 \%$ to $30 \%$ depending on the approach used for diagnosis, and fatal PE occurs in $0.2-0.9 \%$ of patients $[9,10]$. Several independent risk factors for VTE were identified in a gynecological population, including a history of VTE, current diagnosis of gynecologic cancer, increasing age, African American race and prolonged surgical time. The risk of VTE after gynecologic laparoscopic surgery is uncertain. When prescribing antithrombotic prophylaxis before gynecological surgery, additional VTE risk factors must be taken into account such as the extent and duration of the surgery, surgical technique, and type of surgery (hysterectomy, myomectomy) and anesthesia. All women scheduled for gynecological surgery should be assessed in terms of the VTE risk and eligibility to thromboprophylaxis using a validated scoring system and recommended in a given hospital.

The American College of Chest Physicians (ACCP) guidelines incorporated a risk stratification model, the Caprini risk assessment model, in which each independent risk factor is associated with specific points (range from 1 to 5) based on the risk for VTE for each factor (Table 1) [10].

\section{Mechanical prophylactic measures}

Since postoperative thrombi mostly begin within 24 hours after the surgery in the capacitance calf vein. Reduction in DVT formation with this method was observed in $36 \%$ women, although graduated compression stockings were more effective when combined with an additional prophylactic method in women undergoing pharmacological measures [11]. Venous stasis reduction is achieved by regularly compression of calf with tight. When used during and after major gynecologic surgery Intermittent pneumatic compression (IPS) appear to be as effective as low-dose unfractionated heparin (UFH) and low molecular weight heparin (LMWH) in reducing DVT incidence up to 69\% [11]. However, this type of thromboprophylaxis is infrequently used in Polish hospitals.

\section{Low-dose unfractionated heparin (UFH)}

When administered subcutaneously starting 2 hours before surgery and continued every 8-12 hours postoperatively, numerous controlled trials have found low-dose unfractionated heparin effective in preventing VTE. A major concern is the potential for increased perioperative bleeding complications. Moreover, about $6 \%$ of patients receiving this form of prophylaxis will experience heparin-induced thrombocytopenia [12].
Table 1. Caprini risk model (modified, based on [11])

\begin{tabular}{l} 
Age $41-60$ years \\
Swollen legs (current) \\
Varicose veins \\
Obesity (BMI $>25 \mathrm{~kg} / \mathrm{m}^{2}$ ) \\
Minor surgery planned \\
Medical patient currently at bed rest \\
History of prior major surgery (<1 month) \\
Serious lung disease including pneumonia \\
(<1 month) \\
Oral contraceptives or hormone \\
replacement therapy \\
Pregnancy or postpartum (< 1 month) \\
History of unexplained stillborn infant, \\
recurrent spontaneous abortion ( $>3$ ), \\
premature birth with hypoxemia or \\
growth-restricted infant \\
Age $61-74$ \\
Malignancy (present or previous) \\
Laparoscopic surgery ( $>45$ min) \\
Patient confined to bed ( $>72$ h) \\
Central venous access \\
Major surgery ( $>45$ min) \\
Age 75 or older \\
History of DVT/PE \\
Positive factor V Leiden \\
Heparin-induced thrombocytopenia (HIT) \\
Positive Prothrombin $20210 \mathrm{~A}$ \\
Positive lupus anticoagulant \\
Other congenital or acquired \\
thrombophilia \\
Stroke (< 1 month) \\
Multiple trauma (< 1 month) \\
\hline
\end{tabular}
represents 5 points Multiple trauma $(<1$ month $)$

$\mathrm{BMI}$ - body mass index; DVT — deep vein thrombosis; PE — pulmonary embolism

\section{Low molecular weight heparins (LMWH)}

The most commonly used agent in thromboprophylaxis in gynecology is LMWH. Advantages of LMWH over UFH include greater bioavailability and once-daily dosing. The most common side effects are bleeding, allergic reactions and injection side reactions. Moreover, heparin induced thrombocytopenia is very rarely observed with LMWH [12]. However, renal function should be considered when selecting and dosing these agents, because they are excreted by kidneys. A Cochrane review of randomized, controlled trials in gynecologic patients undergoing major surgery and a systematic analysis of gynecologic oncology patients found LMW heparin and low-dose unfractionated heparin equally useful in preventing VTE [12].

\section{Non-vitamin K antagonist oral anticoagulants (NOACs)}

These agents include inhibitors of factor Ila (dabigatran) and factor Xa (rivaroxaban, apixaban and edoxaban). An advantage to their use is that they do not require routine laboratory monitoring and they would be more convenient than $\mathrm{LMWH}$ which requires daily subcutaneous 
Table 2. Risk classification and recommended thromboprophylaxis (based on [17])

\begin{tabular}{|l|l|l|}
\hline Level of VTE risk & Definition & \multicolumn{1}{l}{ Suggested thromboprophylaxis } \\
\hline Low & $\begin{array}{l}\text { Minor surgery (less than } 30 \text { min) or noncomplex laparoscopic } \\
\text { surgery in patients with no additional risk factors }\end{array}$ & Early, frequent ambulation \\
\hline Moderate & $\begin{array}{l}\text { Minor or laparoscopic surgery in patients with additional risk } \\
\text { factors; major gynecologic surgery for benign disease and no } \\
\text { additional risk factors }\end{array}$ & $\begin{array}{l}\text { LMWH or low-dose UFH, } \\
5,000 \text { units twice a day, or IPC or GCS }\end{array}$ \\
\hline High & $\begin{array}{l}\text { Major surgery in patients with additional risk factors; } \\
\text { major surgery in patients with malignancy }\end{array}$ & $\begin{array}{l}\text { LMWH or low-dose UFH } \\
5,000 \text { units three times a day, or IPC } \\
\text { Alternative considerations include a combination } \\
\text { of low-dose UFH or LMWH plus mechanical prophylaxis } \\
\text { with GCS or IPC }\end{array}$ \\
\hline Very high & $\begin{array}{l}\text { Major surgery in patients older than } 60 \text { years with cancer, } \\
\text { a prior venous thromboembolism, or both }\end{array}$ & $\begin{array}{l}5,000 \text { units three times a day, plus IPC or GCP } \\
\text { Consider continuing LMWH prophylaxis for up to 4 weeks } \\
\text { after discharge }\end{array}$
\end{tabular}

LMWH — low molecular weight heparins; UFH — unfractionated heparin; IPC — intermittent pneumatic compression; GCS — graduated compression stockings

administration. However, due to the lack of data confirming their effect on perioperative thromboprophylaxis, these drugs are not approved in patients requiring gynecological surgery [12].

Evidence-based guidelines regarding VTE prophylaxis are available from ACCP and The American College of Obstetrics and Gynecology (ACOG) [3]. All the above guidelines recommend that women with moderate risk of VTE should receive HNF 5000 units twice a day and women with a very high risk of VTE - HNF therapy every $8 \mathrm{~h}$. The ACCP guidelines for gynecological surgery patients at high risk of major bleeding complications were shown in Table 3. Furthermore, in women undergoing gynecological surgery at moderate or high risk for VTE who are at high risk for major perioperative bleeding complications should be given mechanical prophylaxis, preferably with intermittent pneumatic compression (IPC). The ACCP guidelines recommend the use of low-dose aspirin, fondaparinux or mechanical prophylaxis, preferably with IPC before the surgery for women with contraindication to both LMWH and unfractionated heparin treatment. The Polish Gynecological Society has developed guidelines based on recommendations of the American College of Obstetricians and Gynecologists (Table 2) [8]. The Polish guidelines approved by various Polish societies presented by Zawilska et al are based on the 2012 ACCP guidelines which slightly differ from above [13].

When prescribing antithrombic therapy before the surgery it should be also provided the potential risk of perioperative bleeding complications in women threated with antithrombotic therapy due to concomitant diseases such as atrial fibrillation.

Evaluating the extent of the surgery, due to ACCP statement, women undergoing minor surgical or non-invasive procedures do not need to interrupt their antithrombotic therapy. However, to minimize the risk for perioperative bleeding complications patients having major surgical or
Table 3. Dosage of bridging anticoagulation in VTE prevention in gynecological patients (modified, based on [11])

\begin{tabular}{|c|c|}
\hline $\begin{array}{l}\text { Estimated risk for } \\
\text { thromboembolism }\end{array}$ & Bridging anticoagulation \\
\hline High & $\begin{array}{l}\text { "High-dose" (therapeutic-dose): } \\
\text { - enoxaparin } 1 \mathrm{mg} / \mathrm{kg} \text { BID or } 1.5 \mathrm{mg} / \mathrm{kg} \text { QD } \\
\text { - dalteparin } 100 \mathrm{IU} / \mathrm{kg} \text { BID or } 200 \mathrm{IU} / \mathrm{kg} \text { QD } \\
\text { - IV UFH to attain aPTT 1.5- to } 2 \text {-times the } \\
\text { control aPTT }\end{array}$ \\
\hline Moderate & $\begin{array}{l}\text { "Intermediate-dose": } \\
\text { • eg, enoxaparin } 40 \text { mg BID }\end{array}$ \\
\hline Low & $\begin{array}{l}\text { "Low-dose" (prophylactic-dose): } \\
\text { - enoxaparin } 30 \text { mg BID or } 40 \text { mg QD } \\
\text { - dalteparin } 5000 \text { IU QD } \\
\text { - UFH 5000-7500 IU BID }\end{array}$ \\
\hline
\end{tabular}

invasive procedures should have their antithrombic therapy be intermitted (Table 3).

To minimize the risk of perioperative bleeding in women undergoing warfarin, the treatment should be stopped in 5 days before the surgery. However, patients taking antiplatelet agents should interrupt the treatment in 7-10 days before the surgery.

In patients with increased risk for thromboembolism (TE) bridging therapy with heparin should be considered. After surgery, bridging therapy increases hemorrhagic incidents. The risk of perioperative bleeding depends on the anticoagulant dose and proximity to surgery with higher risk if the treatment starts closer to surgery. It has been reported that low-dose LMWH or UFH effectively prevent postoperative VTE, whereas, there is no evidence that this treatment is efficient in acute TE prophylaxis [12]. Furthermore, in women with a mechanical heart valve, atrial fibrillation or VTE at high risk for thromboembolism, the ACCP experts suggest bridging anticoagulation therapy (Table 4).

In resuming antithrombotic therapy, it should be emphasized that the different time period is needed to achieve 
Table 4. ACCP recommendation antithrombotic prophylaxis (based on [11])

\begin{tabular}{|l|l|}
\hline Risk of VTE & Recommendation \\
\hline Very low $(<0.5 \%$, Caprini score, 0$)$ & No specific pharmacologic or mechanical prophylaxis other than early ambulation \\
\hline Low $(1.5 \%$, Caprini score, $1-2)$ & Mechanical prophylaxis, preferably with IPC \\
\hline Moderate $(\sim 3.0 \%$; Caprini score, 3-4) & LMWH, low-dose UFH or mechanical prophylaxis, preferably with IPC \\
\hline High ( 6.0\%; Caprini score, $\geq 5)$ & $\begin{array}{l}\text { Pharmacologic prophylaxis with LMWH or low-dose UFH. The mechanical prophylaxis with elastic } \\
\text { stockings or IPC should be added to pharmacologic prophylaxis }\end{array}$ \\
\hline
\end{tabular}

LMWH - low molecular weight heparins; UFH — unfractionated heparin; IPC — intermittent pneumatic compression

a therapeutic effect after warfarin (2-3 days), LMWH (3-5 h), ASA (minutes), clopidogrel (3-7 days). The ACCP guidelines suggest the VKA treatment should be resumed 12-24 hrs after surgery and when the adequate hemostasis is obtained.

The ACCP guidelines state that most thrombotic or bleeding complications occur within first two weeks after the surgery, therefore strict observation is needed in postoperative women to implement early diagnosis and treatment limiting complications.

\section{THROMBOPROPHYLAXIS IN GYNECOLOGICAL ONCOLOGY PATIENTS}

Patients with cancer have up to 7-fold increase risk of VTE in comparison to the general population [14-16]. It has been reported that approximately $38 \%$ of gynecological oncology patients will suffer from perioperative thromboembolic complications without VTE prophylaxis, and PE remains the leading cause of postoperative death among these patients $[17,18]$. It is due to the fact that patients with active malignancy have additionalVTE risk factors such as the malignancy itself, advanced age, vascular compression by a pelvic mass, lengthy surgery, vascular injury, and treatment with chemotherapy, which increases thrombotic risk [19].

Among gynecological tumors, ovarian cancer is associated with $5-16.6 \%$ risk of VTE and it is one of the highest incidence rates of venous thrombosis [20]. The molecular basis for increased risk of VTE among theese patients is still unknown, however, elevated tissue factor and D-dimer levels are suggested to promote a hypercoagulable state [21]. The VTE risk factors have been divided into early and late depending on the time of thrombembolism onset in relation to the cancer diagnosis. Early thromboembolic events are related to surgery and chemotherapy, whereas later VTE incidences were linked to older age, prior history of DVT, FIGO stage IIC-IV, and presence of residual tumor after initial surgery [22]. Moreover, the risk of VTE depends also on the histological subtypes of carcinomas and for example, the clear cell tumor is associated with the highest VTE incidence of 11-27\% [23].

Endometrial carcinoma is one of the most common gynecologic malignancies. The most prominent risk factors include metabolic syndrome with obesity, hypertension, insulin resistance, diabetes, and dyslipidemia [24]. Recent data have shown that VTE in endometrial cancer is associated with tumor aggressiveness. Moreover, subclinical VTE was observed in relatively large subsets of endometrial cancer patients prior to surgical treatment and more than half of the cases were diagnosed at around the time of recurrence or progression of tumor [25]. So far the relationship between the histological type of uterine cancer and VTE in unclear [26]. However, Matsuo et al. indicated that uterine serous carcinoma (type 2 endometrial cancer) was the most common histologic subtype associated with VTE [23].

The frequency of VTE in patients with diagnosed cervical cancer vary widely from 0-34\% mostly because of heterogeneous patient populations in individual studies and methodological differences [24]. The most common type of cervical cancer is squamous-cell carcinoma, with adenocarcinoma being the second most common. Probably, mucin-producing tumours (what was proved in pancreatic tumours) are associated with a higher risk of VTE [26]. In the cervical cancer, disease stage is defined by the extent of tumour spread to the parametria or pelvic side wall. Therefore, the tumor's proximity to the pelvic veins may result in venous compression and stasis, which are considered to increase the risk of VTE. It was reported that patients with advanced-stage cervical carcinoma have elevated concentrations of von Willebrand factor, fibrinopeptide $A$ and D-dimer levels. Although, the clinical implication of this finding is unknown, the general cancer population show an increased risk of VTE in advanced-stage disease [27].

\section{Treatment-related risk}

In addition to the type and stage of cancer, oncological treatment also modulates the thrombotic potential. Following treatment: surgery, chemotherapy, antiangiogenic drugs, immunomodulatory agents, erythropoiesis-stimulating agents (ESAs), blood transfusions, and central venous catheters are all reported to be associated with an increased risk of VTE.

Major surgical procedures are associated with increased risk of venous thrombosis both in cancer and noncancer patients. In cancer patients, risk of three-months postoperative venous thrombosis is reported to be twice as high as in noncancer patients [27]. The prevalence of DVT in untreated 
gynecologic oncology patients undergoing major surgery is approximately $40 \%$, while the range of fatal PE is between 0.2 and $0.9 \%$ [28]. In patient diagnosed with endometrial or cervical cancer minimally invasive surgery (MIS) is offered for definitive primary treatment. It was reported that this type of surgery is associated with less than $2 \%$ risk ofVTE, which is definitely lower comparing to the open surgery. However, this low rate ofVTE is underestimated due to the fact that patients used mechanical and/or anticoagulant thromboprophylaxis [27].

Chemotherapy leads to hypercoagulable state by secretion of tumor lysis products and endothelial damage. Predictive biomarkers for VTE in ambulatory cancer patients have been proposed but, the utility of them in gynaecological patients has not been well investigated [28]. Recent data from the Vienna prospective study show that elevated levels of $\mathrm{P}$-selectin are predictive of thrombosis, and elevated D-dimer in addition to prothrombin 1.2 fragments $(F 1+2)$ are associated with increased risk ofVTE in patients receiving chemotherapy [29]. Moreover, a prospective study reported 2.7-fold increase in arterial thrombosis, and a 47-fold increase in the mortality rate from VTE in patient undergoing chemotherapy compared to the general population [15].

Radiotherapy has also been reported as a probable risk factor for VTE, but its association with VTE has been poorly studied [30]. An essential component of curative radiotherapy for cervical cancer is intracavitary brachytherapy. It involves instrumentation of the uterus under conscious sedation or general anaesthesia and immobilisation of the patient for hours to plan and deliver treatment. The available data suggest 1-1.2\% incidence of VTE among women treated with brachytherapy for a variety of gynecologic tumors [30].

New anti-angiogenic drugs are entered to the treatment of various solid tumors [31]. However, it was reported that the addition of bevacizumab to chemotherapy regimens in ovarian cancer tratment is associated with a $12 \%$ incidences of VTE. Mechanisms for this side-effect are not well documented and, although bevacizumab has reported activity in cervical cancer it has not been widely studied in this setting [31].

Currently reported data show that supportive care agents, including erythropoietin and darbopoetin as an alternative to red blood cell transfusions for correcting anaemia, appear to increase the risk of VTE and mortality in cancer patients [32].

\section{Thrombophylaxis}

Patients with active cancer disease develop thrombotic complications spontaneously, without any other risk factors. A history of VTE puts patients with cancer at a high risk of recurrence, that is why the systematic use of mechanical or pharmacological prophylaxis should be considered.
Despite of using thromboprophylaxis, some patients still develop VTE after discharge from the hospital. This phenomenon is associated with the fact that VTE prophylaxis is given to most cancer patients only during hospitalization and the treatment is interrupted after discharge due to the risk of bleeding which is more common in cancer patients [17]. Although warfarin remains the mainstay of anticoagulant therapy for VTE treatment, LMWH has been studied extensively and has been adopted as the anticoagulant of choice in the oncology setting [17]. The non vitamin $\mathrm{K}$ oral anticoagulants, i.e. direct thrombin inhibitors and factor-Xa inhibitors, are gaining acceptance as alternative oral anticoagulants to warfarin, they have not yet been sufficiently studied to be recommended for the treatment or prophylaxis of thrombosis in patients with cancer [13]. It has been reported that in oncological patients at higher risk of perioperative bleeding graduated compression stockings and sequential compression devices should be given.

The 2012 ACCP guidelines suggest extended-duration pharmacologic prophylaxis (4 weeks) with LMWH for high-VTE-risk women undergoing gynecological surgery for cancer who are not otherwise at high risk for major bleeding complications [15]. However, ACCP states that further clinical trials are required to elaborate antithrombotic prophylaxis in gynecological oncology patients. The Polish Gynecological Society suggests to proceed these patients like in women with a very high risk of VTE (Table 2).

It has been reported that open gynecologic surgeries should be supported by the use of VTE prophylaxis. Despite the fact that the necessity of thromboprophylaxis in MIS is unclear recommendation stated by various medical organizations including ACOG for patients undergoing laparoscopy are the same as for women undergoing laparotomy. To our knowledge, among large societies, the ACCP is the only one that recommends against routine thromboprophylaxis for gynecology patients undergoing MIS, unless additional VTE risk factors, such as cancer are present. At our site, we have different procedures according to the duration of hospitalization. In women prepared in the early recovery after surgery (ERAS) protocol and undergoing laparoscopic gynecologic oncology procedures we practice the same-day discharge after admission and they do not receive any form of VTE prophylaxis. On the other hand, women with current malignancy, which we expect to extend the stay in hospital we follow according the Polish Gynecological Society recommendation.

\section{PROPHYLAXIS IN WOMEN UNDERGOING ASSISTED REPRODUCTIVE TECHNIQUES (ART)}

Assisted reproduction appears to be associated with an increased risk of venous thromboembolism (OR: 4.3, 95\% Cl: 2.0-9.4) [33]. The mechanism behind the increased frequency of VTE associated with ART remains unknown [33]. 
Increased estrogen level may induce a procoagulant effect by increasing levels of von Willenbrand factor, factor VIII, factor $\mathrm{V}$, and fibrinogen, together with decreased levels of the anticoagulants protein S and antithrombin [34]. However, the clinical relevance of these changes is unclear because most variables remain within the normal range [19]. It has been suggested that women with failure of assisted reproduction are more often positive for the factor $V$ Leiden mutation and antiphospholipid antibodies [34]. However, these results were not confirmed in a meta-analysis of cohort studies [33].

The most serious complication of induction of ovulation used in ART is ovarian hyperstimulation syndrome (OHSS). The frequency and severity of OHSS depend on the clinical condition and the etiology in which ovulation is needed. The incidence and severity of OHSS vary with the different clinical conditions in which ovulation is induced and the type of the protocol which is used for ovarian stimulation. In women with anovulatory cycles the frequency of mild OHSS is $5-10 \%$. However, $2-4 \%$ women treated by the ART suffers moderate OHSS and 0.1-0.5\% - severe OHSS [35].

The vascular permeability is associated with the release of ovary vasoactive substances, such as vascular endothelial growth factor (VEGF), angiotensine, histamine, insulin-like growth factor 1 (IGF-1), epidermal growth factor (EGF), interleukins. It results in fluid translocation out of the blood vessels and it leads to to hypovolemia and hemostasis disorders [33].

Low-dose ASA and/or prophylactic doses of LMWH are prescribed to women approaching ART. ASA treatment is thought to have positive effects both on placental circulation, which leads to better implantation with higher pregnancy rate and on enhancing ovarian response to stimulation [33]. In turn, heparin is considered to improve implantation rates by both reducing the risk of implantation site microthrombosis and improving endometrial receptivity with decidualization of endometrial stromal cells, as well as trophoblast adhesion, and invasiveness [22]. However, available clinical data are inconsistent [35].

The ACCP guidelines do not recommend any thromboprophylaxis for women undergoing assisted reproduction. However, women with developed OHSS should receive prophylactic LMWH for 3 months after recovery regardless the success of ART. From our experience, we do not use routine thromboprophylaxis due to the risk of bleeding during ovarian puncture in the course of the in vitro procedure.

\section{CONCLUSIONS}

Prophylaxis in gynecological patients and women undergoing assisted reproductive techniques should be chosen individually for the patient, taking into account the possible risk factors for VTE and perioperative bleeding complications. Too small dose and inadequate treatment period increases the risk of developing the disease in the most dangerous complications - pulmonary embolism. Due to the possibility of bleeding complications enhanced surveillance and monitoring therapy especially after surgery is needed. However, prevention of venous thromboembolism in cancer is an important challenge, because patients experiencing a thrombotic episode have a poor outcome with greater probability of death.

The decision for duration of thromboprophylaxis should be taken by balancing the risk of VTE and risk of bleeding. Factors that should be considered for making the decision are the incidence and burden of the VTE. Standardized and widely implemented approach to thromboprophylaxis in each gynecology center is needed to optimize clinical outcomes.

\section{REFERENCES}

1. Heit JA. Epidemiology of venous thromboembolism. Nat Rev Cardiol. 2015, 12, 464-474.

2. Corr BR, Winter AM, Sammel MD, [et al.]. Effectiveness and safety of expanded perioperative thromboprophylaxis in complex gynecologic surgery. Gynecol Oncol. 2015, 3, 501-506.

3. Dentali F, Cei M, Mumoli N, [et al.]. How to predict short- and long-term mortality in patients with pulmonary embolism? Pol Arch Med Wewn. 2015, 125, 82-88.

4. Rahn DD, Mamik MM, Sanses TV, [et al.]. Venous thromboembolism prophylaxis in gynecologic surgery: a systematic review. Obstet Gynecol. 2011, 118, 1111-1125.

5. Kyrle PA. Venous thrombosis: who should be screened for thrombophilia in 2014? Pol Arch Med Wewn. 2014, 124, 65-69.

6. Trusz-Gluza M, Filipecki A, Urbańczyk-Świć D. Patients with atrial fibrillation and low risk of stroke: do they really need anticoagulation? Pol Arch Med Wewn. 2015, 125, 678-684.

7. Tafur A, Douketis JD. Perioperative anticoagulant management in patients with atrial fibrillation: practical implications of recentclinical trials. Pol Arch Med Wewn. 2015, 125, 666-671.

8. Low-weight heparin treatment in obstetrics and gynecology - the Polish Gynecological Society. Ginekol Pol. 2010, 81, 311-314.

9. Undas A, Brummel-Ziedins K, Mann KG. Why does aspirin decrease the risk of venous thromboembolism? On old and novel antithrombotic effects of acetyl salicylic acid. J Thromb Haemost. 2014, 12, 1776-1787.

10. Kearon C, AkI EA, Ornelas J, [et al.]. Antithrombotic Therapy for VTE Disease: CHEST Guideline and Expert Panel Report. Chest. 2016, 149, 315-352.

11. Gould MK, Garcia DA, Wren SM, [et al.]. American College of Chest Physicians. Prevention of VTE in nonorthopedic surgical patients: Antithrombotic Therapy and Prevention of Thrombosis, 9th ed: American College of Chest Physicians Evidence-Based Clinical Practice Guidelines. Chest. 2012, 14, 227S-277S.

12. Mahe I, Aghassarian $M$, Drouet $L$, [et al.]. Tinzaparin and enoxaparin given at prophylactic dose for eight days in medical elderly patients with impaired renal function: a comparative pharmacokinetic study. Thromb Haemost. 2007, 97, 581-586.

13. den Exter PL, Kooiman J, van der Hulle T, [et al.]. New anticoagulants in the treatment of patients with cancer-associated venous thromboembolism. Best Pract Res Clin Haematol. 2013, 26, 163-169.

14. Zawilska K, Bała MM, Błędowski P. Polish guidelines for the prevention and treatment of venous thromboembolism. 2012 update. Pol Arch Med Wewn. 2012, suppl 2, 73-74.

15. Clarke-Pearson DL, Abaid LN. Prevention of venous thromboembolic events after gynecologic surgery. Obstet Gynecol. 2012, 119, 155-167.

16. Khorana AA, Francis CW, Culakova E, [et al.]. Frequency, risk factors, and trends for venous thromboembolism among hospitalized cancer patients. Cancer. 2007; $110,2339-2346$.

17. Stroud W, Whitworth JM, Miklic M, [et al.]. Validation of a venous thrombembolism risk assessment model in gynecologic oncology. Gynecol Oncol. 2014, 134, 160-163.

18. Lyman GH, Khorana AA, Falanga A, [et al.]. American Society of Clinical Oncology guideline: recommendations for venous thromboembolism prophylaxis and treatment in patients with cancer. J Clin Oncol. 2007, $25,5490-5505$. 
19. Barber EL, Clarke-Pearson DL. The limited utility of currently available venous thromboembolism risk assessment tools in gynecological oncology patients. Am J Obstet Gynecol. 2016 [ahead of print].

20. Schmeler KM, Wilson GL, Cain K. Venous thromboembolism (VTE) rates following the implementation of extended duration prophylaxis for patients undergoing surgery for gynecologic malignancies. Gynecol Oncol. 2013, 128, 204-208.

21. Duska LR, Garrett L, Henretta M, [et al.]. When 'never-events' occur despite adherence to clinical guidelines: the case of venous thromboembolism in clear cell cancer of the ovary compared with other epithelial histologic subtypes. Gynecol Oncol. 2010, 116, 374-377.

22. Xiang-Hua Y, Hong-Yan J, Xiao-Rui X, [et al.]. Clinical analysis of endometrial cancer patients with obesity, diabetes, and hypertension. Int J Clin Exp Med. 2014, 7, 736-743.

23. Matsuo K, Yessaian AA, Lin YG, [et al.]. Predictive model of venous thromboembolism in endometrial cancer. Gynecol Oncol. 2011, 128, 544-551.

24. Satoh T, Oki A, Uno K, [et al.]. High incidence of silent venous thromboembolism before treatment in ovarian cancer. Br J Cancer. 2007, 97, 1053-1057.

25. Sedlis A, Bundy BN, Rotman MZ, [et al.]. A randomized trial of pelvic radiation therapy versus no further therapy in selected patients with stage IB carcinoma of the cervix after radical hysterectomy and pelvic lymphadenectomy: a Gynecologic Oncology Group Study. Gynecol Oncol. 1999, 73, 177-183.

26. Nguyen NT, Hinojosa MW, Fayad C, [et al.]. Laparoscopic surgery is associated with a lower incidence of venous thromboembolism compared with open surgery Annals of Surgery. 2011, 6, 1021-1027.
27. Bouchard-Fortier G, Geerts WH, Covens A, [et al.]. Is venous thromboprophylaxis necessary in patients undergoing minimally invasive surgery for a gynecologic malignancy? Gynecol Oncol. 2014, 134, 228-232.

28. Ay C, Vormittag R, Dunkler D, [et al.]. D-dimer and prothrombin fragment $1+2$ predict venous thromboembolism in patients with cancer: results from the Vienna Cancer and Thrombosis Study. J Clin Oncol. 2009, 27, 4124-4129.

29. Matzdorff A, Ledig B, Stuecker M, Riess H. Practice Patterns for Prophylaxis and Treatment of Venous Thromboembolism in German Cancer Patients. Oncol Res Treat. 2016, 39, 194-201.

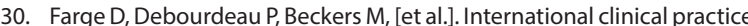
guidelines for the treatment and prophylaxis of venous thromboembolism in patients with cancer. J Thromb Haemost. 2013, 11, 56-70.

31. Nalluri SR, Chu D, Keresztes R, [et al.]. Risk of venous thromboembolism with the angiogenesis inhibitor bevacizumab in cancer patients: a meta-analysis. JAMA. 2008, 300, 2277-2285.

32. Bennett CL, Silver SM, Djulbegovic B, [et al.]. Venous thromboembolism and mortality associated with recombinant erythropoietin and darbepoetin administration for the treatment of cancer-associated anemia. JAMA. 2008, 299, 914-924.

33. Jacobsen AF, Skjeldestad FE, Sandset PM. Ante- and postnatal risk factors of venous thrombosis: a hospital-based case-control study. J Thromb Haemost. 2008, 6, 905-912.

34. ESHRE Capri Workshop Group. Venous thromboembolism in women: a specific reproductive risk. Hum Reprod Update. 2013, 19, 471-482.

35. Mor YS, Schenker JG. Ovarian hyperstimulation syndrome and thrombotic events. Am J Reprod Immunol. 2014, 72, 541-548. 